those with a neurological exam documented, no infant had all components of the standardised exam included in the documentation (level of consciousness, activity, tone, posture, primitive reflexes, and autonomic function), preventing retrospective assessment of grade of NE. There was no difference in the quality of documentation between those that did and did not receive $\mathrm{TH}$.

By the time of discharge documentation had improved, and the grade of encephalopathy was detailed in twenty five patients (81\%).

Conclusion The documentation of the neurological examination when determining eligibility for $\mathrm{TH}$ is inadequate. Though the documentation improves by discharge, initial documentation must be improved to ensure appropriate clinical practice and patient safety. To achieve this, educational sessions for staff and trainees has been initiated, after which repeated audit will be conducted as part of this quality improvement initiative.

\section{GP110 PASSING THE BATON- MEDICAL ON CALL TEAM EVENING HANDOVER IN OUR LADIES CHILDREN HOSPITAL CRUMLIN}

Rory Mannion*, Suzanne Kelleher. Our Ladies Children Hospital Crumlin, Dublin 12, Ireland

\subsection{6/archdischild-2019-epa.175}

Background Optimal clinical handover is necessary to improve clinical safety and reduce patient risk. Clinical handover in Our Lady's Children's Hospital Crumlin (OLCHC) occurs at the beginning and end of the on call shift. Clinical handover should be conducted face to face where possible, be conducted verbally using a standardised communication tool, and be supported with relevant, accurate and up-to-date documentation.

Aims To have a member of each subspecialty team attend evening handover and communicate patients who are anticipated to require medical review on call. For each healthcare worker attending handover to sign in attendance sheet. To handover patients using Identification Situation Background Assessment Recommendation (ISBAR) format.

Methods We collected data over a week-day period between $17 / 09 / 2018$ until $28 / 09 / 2018$ in OLCHC. This is a quality improvement project. Data was collected and documented from evening handovers. The following outcome measures were included; subspecialty attendance e.g. doctor from cardiology, respiratory, PICU etc sick patients handed over expected transfers handed over and whether ISBAR format had been used

At the subsequent morning handover patient issues requiring medical review on call were recorded in order to identify patients who should have been highlighted to on call medical team.

Results Over the ten days attendance was recorded at the morning and evening clinical handover. The number of subspecialty teams attending each evening handover is represented here.

\begin{tabular}{lllllllllll}
\hline (Day) & 1 & 2 & 3 & 4 & 5 & 6 & 7 & 8 & 9 & 10 \\
\hline (Number of teams) & 4 & 2 & 5 & 0 & 5 & 0 & 1 & 0 & 4 & 6 \\
\hline
\end{tabular}

ISBAR format was used $56 \%$ of the time by the subspecialty team.
Several clinical risks represented above were not handed over to medical team on call:

- 1st night an unwell patient with cystic fibrosis

- 2nd night an infant with trisomy 21 and a serious congenital anomaly was admitted from a hospital transfer

- 6th night a patient was discharged from PICU

- 7th night two emergency cardiac transfers

- 10th night a seriously unwell patient on dialysis

Discussion There was incomplete attendance of subspecialties at clinical handover. ISBAR was frequently not used. There is scope for improvement in attendance and handover of subspecialty patients. In an attempt to make improvements to clinical handover we have begun to send a text message reminding all non-consultant hospital doctors to attend clinical handover and handover patients. We are in the process of collecting data to assess for improvement.

\section{GP111 INCIDENT REPORTING AND THE NON-CONSULTANT HOSPITAL DOCTOR IN A GENERAL HOSPITAL}

Niofa Canty*, M Elbadry, B Reidy, H Stokes, Michael B O'Neill. Mayo University Hospital, Castlebar, Ireland

\subsection{6/archdischild-2019-epa.176}

Aim and background Incident reporting though poorly named is one of the pillars are of patient safety. Non-Consultant Hospital Doctors (NCHDs), while actively involved in patient care contribute minimally to incident reporting overall. This study evaluated NCHD knowledge of the incident reporting process and undertook an education module

Methods All NCHDS at Mayo University Hospital were invited to participate. Three scenarios were devised, reflective of actual cases, in conjunction with the authors and the quality improvement department. These cases were weighed and considered by the NCHD committee to ascertain clinical relevance for the trainees. A formal small group 30-minute instructional lecture with case discussion and actual case inputting was developed lead by the quality improvement team. Pre and post-test questionnaires on the reporting process were devised that had a direct relevance and trainee perceptions on the training process and barriers to reporting were elicited through thematic evaluation of the post training discussions which were recorded.

Results Forty (48\%) trainees volunteered 23 SHO and 17 Registrars. The mean knowledge scores were 52 pre-test and 67 post-test. Trainees uniformly enjoyed being 'walked through' the reporting process and actually having a test opportunity to complete made it more real. Thematic evaluation suggest that trainees are not clear as to what constitutes an incident, have misconceptions of the incident reporting process and operate in an environment that is not conducive to reporting. Patient safety and incident reporting are not viewed as being interconnected.

Conclusions This educational module enhanced trainee knowledge, corrected misperceptions on its role and suggests that they are more likely to report on clinical incidents. The reporting tool, however, is not geared to the end user which is a hinderance in reporting and the language used facilitates a negative connotation. 